\title{
Interplay of neutrino spin and three-flavour oscillations in a magnetic field
}

\author{
Alexey Lichkunov, ${ }^{a}$ Artem Popov ${ }^{a, *}$ and Alexander Studenikin ${ }^{a, b}$ \\ ${ }^{a}$ Faculty of Physics, Lomonosov Moscow State University, Moscow 119991, Russia \\ ${ }^{b}$ Joint Institute for Nuclear Research, Dubna 141980, Moscow Region, Russia \\ E-mail: ar.popov@physics.msu.ru, zon53@mail.ru, studenik@srd.sinp.msu.ru
}

We develop the approach to the problem of neutrino oscillations in a magnetic field introduced in [1] and extend it to the case of three neutrino generations. The theoretical framework suitable for computation of the Dirac neutrino spin, flavour and spin-flavour oscillations probabilities in a magnetic field is given. The closed analytic expressions for the probabilities of oscillations are obtained accounting for the normal and inverted hierarchies and the possible effect of CP violation. In general, the neutrino oscillation probabilities exhibit quite a complicated interplay of oscillations on the magnetic $\omega_{B}=\mu_{v} B$ and vacuum $\omega_{v a c}=\frac{\Delta m_{i k}^{2}}{4 p}$ frequencies. The obtained results are of interest in applications to neutrino oscillations under the influence of extreme astrophysical environments, for example peculiar to magnetars and supernovas, as well as in studying neutrino propagation in interstellar magnetic fields (see [2]).

*** The European Physical Society Conference on High Energy Physics (EPS-HEP2021), ***

*** 26-30 July $2021 * * *$

*** Online conference, jointly organized by Universität Hamburg and the research center DESY ***

\footnotetext{
${ }^{*}$ Speaker
} 


\section{Neutrino flavour oscillations in a magnetic field}

In this section we briefly describe the approach to derivation of the neutrino oscillations probabilities in a magnetic field developed in [1].

The wave function $v_{i}^{s}(s= \pm 1)$ of a massive neutrino that propagates along $\mathbf{n}_{\mathbf{z}}$ direction in the presence of a constant and homogeneous arbitrary orientated magnetic field can be found as the solution of the following system of Dirac equations in the massive neutrino basis

$$
\left(\gamma_{\mu} p^{\mu}-m_{i}-\mu_{i} \mathbf{\Sigma} \mathbf{B}\right) v_{i}^{s}(p)=0
$$

where $\mu_{i}$ are the neutrino magnetic moments, $i=1,2,3$ and $s= \pm 1$ is a spin number.

This equation can be rewritten in the equivalent Hamiltonian form

$$
\hat{H}_{i} v_{i}^{s}=E v_{i}^{s},
$$

where the Hamiltonian is given by

$$
\hat{H}_{i}=m_{i} \gamma_{0}+\gamma_{0} \boldsymbol{\gamma} \boldsymbol{p}+\mu_{i} \gamma_{0} \boldsymbol{\Sigma} \boldsymbol{B} .
$$

Given the ultrarelativistic approximation, for the neutrino energy spectrum we obtain

$$
E_{i}^{s} \approx p+\frac{m_{i}^{2}}{2 p}+\mu_{i} s B_{\perp}
$$

Since the oscillations lengths are determined by the energy differences $E_{i}^{s}-E_{k}^{s^{\prime}}$, we can expect that the probabilities of neutrino oscillations in a magnetic field exhibit a complex interplay of the oscillations on the vacuum frequencies $\omega_{v a c}=\frac{\Delta m_{i k}^{2}}{4 p}$ and magnetic frequencies $\omega_{B}=\mu_{i} B_{\perp}$.

To classify the neutrino stationary states in a magnetic field we use the generalized spin operator that commutes with the Hamiltonian (3) and can be chosen in the form

$$
\hat{S}_{i}=\frac{m_{i}}{\sqrt{m_{i}^{2} \boldsymbol{B}^{2}+\boldsymbol{p}^{2} B_{\perp}^{2}}}\left[\boldsymbol{\Sigma} \boldsymbol{B}-\frac{i}{m_{i}} \gamma_{0} \gamma_{5}[\boldsymbol{\Sigma} \times \boldsymbol{p}] \boldsymbol{B}\right] .
$$

For the neutrino stationary states the following relations hold:

$$
\begin{gathered}
\hat{S}_{i}\left|v_{i}^{s}\right\rangle=s\left|v_{i}^{s}\right\rangle, s= \pm 1, \\
\left\langle v_{i}^{s} \mid v_{k}^{s^{\prime}}\right\rangle=\delta_{i k} \delta_{s s^{\prime}} .
\end{gathered}
$$

Now in order to solve the problem of the neutrino flavour, spin and spin-flavour oscillations in the magnetic field we expand the neutrino chiral states over the neutrino stationary states:

$$
\begin{aligned}
& v_{i}^{L}(t)=c_{i}^{+} v_{i}^{+}(t)+c_{i}^{-} v_{i}^{-}(t), \\
& v_{i}^{R}(t)=d_{i}^{+} v_{i}^{+}(t)+d_{i}^{-} v_{i}^{-}(t),
\end{aligned}
$$

where $c_{i}^{ \pm}$and $d_{i}^{ \pm}$do not depend on time. For the coefficients $c_{i}^{+(-)}$and $d_{i}^{+(-)}$it is just straightforward

$$
\left|c_{i}^{ \pm}\right|^{2}=\left\langle v^{L}\left|\hat{P}_{i}^{ \pm}\right| v^{L}\right\rangle,\left|d_{i}^{ \pm}\right|^{2}=\left\langle v^{R}\left|\hat{P}_{i}^{ \pm}\right| v^{R}\right\rangle
$$


where

$$
\hat{P}_{i}^{ \pm}=\frac{1 \pm \hat{S}_{i}}{2}
$$

are the projection operators. The explicit expressions for the coefficients (10) can be found in [1].

Finally, we calculate the probabilities of flavour oscillations in a magnetic field:

$$
\begin{array}{r}
P_{v_{e}^{L} \rightarrow v_{\alpha}^{L}}(L)=\left|\left\langle v_{\alpha}^{L} \mid v_{e}^{L}(L)\right\rangle\right|^{2}=\mid\left(\left|c_{1}^{+}\right|^{2} e^{-i E_{1}^{+} L}+\left|c_{1}^{-}\right|^{2} e^{-i E_{1}^{-} L}\right) U_{11} U_{\alpha 1}^{*}+ \\
\left(\left|c_{2}^{+}\right|^{2} e^{-i E_{2}^{+} L}+\left|c_{2}^{-}\right|^{2} e^{-i E_{2}^{-} L}\right) U_{12} U_{\alpha 2}^{*}+\left.\left(\left|c_{3}^{+}\right|^{2} e^{-i E_{3}^{+} L}+\left|c_{3}^{-}\right|^{2} e^{-i E_{3}^{-} L}\right) U_{13} U_{\alpha 3}^{*}\right|^{2}
\end{array}
$$

where $\alpha=e, \mu, \tau$ is neutrino flavour and $L$ is the distance travelled by a neutrino.

\section{Cosmic neutrino oscillations}

In this section we consider ultrahigh-energy cosmic neutrinos propagation through the interstellar magnetic field and show that the oscillations described above can modify the neutrino fluxes coming form astrophysical objects. It was measured by the Interstellar Boundary Explorer that the strength of the interstellar magnetic field is approximately $2.9 \mu \mathrm{G}$. In our analysis for simplicity we assume that $\mu_{1}=\mu_{2}=\mu_{3}=\mu$ and fix the values of the neutrino magnetic moments to be $\mu=10^{-12} \mu_{B}$.

Fig. 1a shows the probabilities of $v_{e} \rightarrow v_{e}, v_{e} \rightarrow v_{\mu}$ and $v_{e} \rightarrow v_{\tau}$ oscillations as a function of the distance $L$ (in parsec) for the case of the neutrino energy equal to $10 \mathrm{ZeV}$. The probabilities indeed exhibit a complicated interplay of oscillations on the following three frequencies: two vacuum frequencies $\omega_{12}=\Delta m_{12}^{2} / 4 p$ and $\omega_{13}=\Delta m_{13}^{2} / 4 p$, and the magnetic frequency $\omega_{B}=\mu B$. Using the indicated above values for the neutrino magnetic moment and for the strength of the magnetic field, and the existed data on $\Delta m_{12}^{2}$ and $\Delta m_{13}^{2}$ one obtains estimations for the corresponding oscillation lengths: $L_{B} \approx 0.4 p c, L_{12} \approx 100 p c$ and $L_{13} \approx 3 p c$.

Fig. $1 \mathrm{~b}$ shows the probability of oscillations into a righthanded neutrino state as a function of the distance $L$ (in parsec). Unlike the probabilities of flavour transitions, the probability of spin oscillations is described by a simple expression

$$
P_{v_{e}^{L} \rightarrow v^{R}}=P_{v_{e}^{L} \rightarrow v_{e}^{R}}+P_{v_{e}^{L} \rightarrow v_{\mu}^{R}}+P_{v_{e}^{L} \rightarrow v_{\tau}^{R}}=\sin ^{2} \mu B L .
$$

Since cosmic neutrinos travel long distances, we can expect that due to the effect of the wavepacket separation the neutrino fluxes on the Earth can be computed using the distant-averaged probabilities. The effects of a magnetic field induce additional modes in the oscillations probabilities and clearly affect the averaged probabilities. Thus, the presence of large enough values of the neutrino magnetic moments can significantly modify the neutrino fluxes measured in the terrestrial experiments.

\section{Conclusion}

We extended our previous results [1] on two-flavour neutrino oscillations in magnetic fields to the case of the three neutrino flavours. It is shown that the probabilities of neutrino flavour oscillations in a magnetic field exhibit quiet complicated dependence of both vacuum and magnetic 
a)

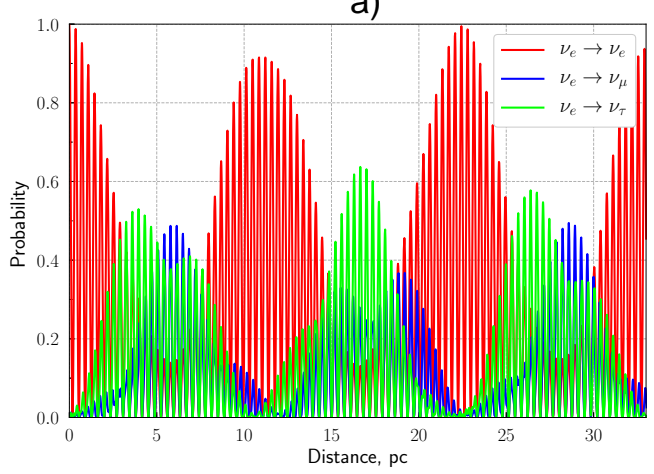

b)

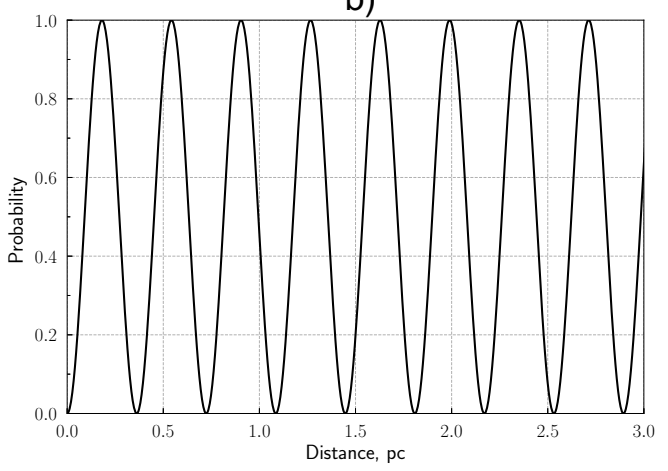

Figure 1: The probabilities of neutrino oscillations in interstellar magnetic field as functions of distance in parsec for the case of neutrino energy equal to $10 \mathrm{ZeV}$. a) Neutrino flavour oscillations $v_{e}^{L} \rightarrow v_{e}^{L}, v_{e}^{L} \rightarrow v_{\mu}^{L}$ and $v_{e}^{L} \rightarrow v_{\tau}^{L}$; b) Neutrino spin oscillations $v_{e}^{L} \rightarrow v^{R}$.

field frequencies. The phenomenon of neutrino oscillations in a magnetic field considered above may significantly modify neutrino fluxes detected on the Earth. A more detailed study of the effect of three flavour neutrino oscillations in a magnetic field will be presented in a forthcoming paper.

The work is supported by the Russian Foundation for Basic Research under grant No. 2052-53022-GFEN-a. The work of A.P. is supported by the Foundation for the Advancement of Theoretical Physics and Mathematics BASIS under grant No. 19-2-6-209-1.

\section{References}

[1] A. Popov and A. Studenikin, Eur. Phys. J. C 79 (2019) no.2, 144 doi:10.1140/epjc/s10052019-6657-z [http://arXiv:1902.08195 [hep-ph]].

[2] P. Kurashvili, K. Kouzakov, L. Chotorlishvili and A. Studenikin, Phys. Rev. D 96 (2017) no.10, 103017 doi:10.1103/PhysRevD.96.103017 [arXiv:1711.04303 [hep-ph]]. 